Burlington, Vermont 05405, USA. Phone: (802) 656-2500; Fax: (802) 656-4523; E-mail: Mark.Nelson@uvm.edu.

1. Lifton, R.P., Gharavi, A.G., and Geller, D.S. 2001 Molecular mechanisms of human hypertension. Cell. 104:545-556.

2. Huang, P.L., et al. 1995. Hypertension in mice lacking the gene for endothelial nitric oxide synthase. Nature. 377:196-197.

3. Shesely, E.G., et al. 1996. Elevated blood pressures in mice lacking endothelial nitric oxide synthase. Proc. Natl. Acad. Sci. U. S. A. 93:13176-13181.

4. Pfeifer, A., et al. 1998. Defective smooth muscle regulation in cGMP kinase I-deficient mice. $E M B O J$. 17:3045-3051.

5. Taylor, M.S., et al. 2003. Altered expression of smallconductance $\mathrm{Ca}^{2+}$-activated $\mathrm{K}^{+}$(SK3) channels modulates arterial tone and blood pressure. Circ. Res. 93:124-131.

6. Brenner, R., et al. 2000. Vasoregulation by the $\beta 1$ subunit of the calcium-activated potassium channel. Nature. 407:870-876.

7. Pluger, S., et al. 2000. Mice with disrupted BK channel $\beta_{1}$ subunit gene feature abnormal $\mathrm{Ca}^{2+}$ spark/STOC coupling and elevated blood pressure. Circ. Res. 87:E53-E60.

8. Gollasch, M., et al. 2002. The BK channel $\beta 1$ subunit gene is associated with human baroreflex and blood pressure regulation. J. Hypertens. 20:927-933.

9. Knaus, H.G., et al. 1994. Primary sequence and immunological characterization of $\beta$-subunit of high conductance $\mathrm{Ca}^{2+}$-activated $\mathrm{K}^{+}$channel from smooth muscle. J. Biol. Chem. 269:17274-17278.

10. Jiang, Z., Wallner, M., Meera, P., and Toro, L. 1999.
Human and rodent MaxiK channel $\beta$-subunit genes: cloning and characterization. Genomics. 55:57-67.

11. Cox, D.H., and Aldrich, R.W. 2000. Role of the $\beta 1$ subunit in large-conductance $\mathrm{Ca}^{2+}$-activated $\mathrm{K}^{+}$ channel gating energetics: mechanisms of enhanced $\mathrm{Ca}^{2+}$ sensitivity. J. Gen. Physiol. 116:411-432.

12. Nelson, M.T., et al. 1995. Relaxation of arterial smooth muscle by calcium sparks. Science. 270:633-637.

13. Perez, G.J., Bonev, A.D., Patlak, J.B., and Nelson, M.T. 1999. Functional coupling of ryanodine receptors to $\mathrm{K}_{\mathrm{Ca}}$ channels in smooth muscle cells from rat cerebral arteries. J. Gen. Physiol. 113:229-238.

14. Jaggar, J.H., Porter, V.A., Lederer, W.J., and Nelson, M.T. 2000. Calcium sparks in smooth muscle. Am. J. Physiol. 278:C235-C256.

15. Zhuge, R., Fogarty, K.E., Tuft, R.A., and Walsh, J.V., Jr. 2002. Spontaneous transient outward currents arise from microdomains where BK channels are exposed to a mean $\mathrm{Ca}^{2+}$ concentration on the order of $10 \mu \mathrm{M}$ during a $\mathrm{Ca}^{2+}$ spark. J. Gen. Physiol. 120:15-27.

16. Knot, H.J., Standen, N.B., and Nelson, M.T. 1998 Ryanodine receptors regulate arterial diameter and wall $\left[\mathrm{Ca}^{2+}\right]$ in cerebral arteries of rat via $\mathrm{Ca}^{2+}$-dependent $\mathrm{K}^{+}$channels. J. Physiol. 508:211-221.

17. Standen, N.B., and Quayle, J.M. 1998. K+ channel modulation in arterial smooth muscle. Acta Physiol. Scand. 164:549-557.

18. Alioua, A., et al. 1998. The large conductance, voltage-dependent, and calcium-sensitive $\mathrm{K}^{+}$channel, Hslo, is a target of cGMP-dependent protein kinase phosphorylation in vivo. J. Biol. Chem. 273:32950-32956.

19. Hofmann, F., Ammendola, A., and Schlossmann, J.
2000. Rising behind NO: cGMP-dependent protein kinases. J. Cell Sci. 113:1671-1676.

20. McManus, O.B., et al. 1995. Functional role of the $\beta$ subunit of high conductance calcium-activated potassium channels. Neuron. 14:645-650.

21. Meera, P., Wallner, M., Jiang, Z., and Toro, L. 1996. A calcium switch for the functional coupling between $\alpha$ (hslo) and $\beta$ subunits (KV,Ca beta) of maxi K channels. FEBS Lett. 382:84-88.

22. Cox, D.H., Cui, J., and Aldrich, R.W. 1997. Allosteric gating of a large conductance Ca-activated $\mathrm{K}^{+}$ channel. J. Gen. Physiol. 110:257-281.

23. Amberg, G.C., Bonev, A.D., Rossow, C.F., Nelson, M.T., and Santana, L.F. 2003. Modulation of the molecular composition of large conductance, $\mathrm{Ca}^{2+}$ activated $\mathrm{K}^{+}$channels in vascular smooth muscle during hypertension. J. Clin. Invest. 112:717-724. doi:10.1172/JCI200318684.

24. Amberg, G.C., and Santana, L.F. 2003. Downregulation of the BK channel $\beta_{1}$ subunit in genetic hypertension. Circ. Res. 93:965-971.

25. Valverde, M.A., et al. 1999. Acute activation of Maxi$\mathrm{K}$ channels (hSlo) by estradiol binding to the $\beta$ subunit. Science. 285:1929-1931.

26. Kotlikoff, M., and Hall, I. 2003. Hypertension: $\beta$ testing. J. Clin. Invest. 112:654-656. doi:10.1172/ JCI200319580.

27. Fernández-Fernández, J.M., et al. 2004. Gain-offunction mutation in the KCNMB1 potassium channel subunit is associated with low prevalence of diastolic hypertension. J. Clin. Invest. 113:1032-1039. doi:10.1172/JCI200420347.

28. Horrigan, F.T., and Aldrich, R.W. 2003. Coupling between voltage sensor activation, $\mathrm{Ca}^{2+}$ binding and channel opening in large conductance (BK) potassium channels. J. Gen. Physiol. 120:267-305.

\title{
Functional obstruction: the renal pelvis rules
}

\author{
Cathy Mendelsohn
}

\author{
Departments of Urology and Pathology, Columbia University College of Physicians and Surgeons, New York, New York, USA.
}

\begin{abstract}
Failure in the peristaltic mechanism that conducts urine from the kidney to the bladder can lead to hydronephrosis, a common birth defect associated with obstructive nephropathy. New animal models reveal molecular pathways important for peristalsis and point to the central role of the renal pelvis in urine transport (see the related article beginning on page 1051).
\end{abstract}

Hydronephrosis, enlargement of a kidney as a result of urine collection in the renal pelvis or calyces, is present in about $1 \%$ of newborns and can lead to obstructive nephropathy $(1,2)$. Often caused by static anatomic occlusion (e.g., by stones) or by failure of the peristaltic mechanism, the underlying genetic and cellular defects at play in obstructive nephropathy are not well understood. Urine is transported out of the papilla by a peristaltic process to the

Nonstandard abbreviations used: cytoplasmic nuclear factor of activated T cells (NFATc).

Conflict of interest: The author has declared that no conflict of interest exists.

Citation for this article:

J. Clin. Invest. 113:957-959 (2004).

doi:10.1172/JCI200421402. pelvis and ureters and then is stored in the bladder. Peristalsis is initiated in the renal pelvis and is propagated along the urinary tract by smooth muscle cells in the ureter coat. Hydronephrosis is associated with a number of congenital abnormalities including vesico-ureteral reflux and hydroureter, which can be caused by physical obstruction. Despite their different appearances, these malformations most likely stem from a common defect: failure of ureters to join the bladder properly (Figure 1A) (3). Ectopically terminating ureters can join the bladder outside the normal position in the trigone or can join the sex ducts or urethra or end blindly. However, in many congenital cases of hydronephrosis or hydroureter, no physical obstruction can be demonstrated (Figure 1B). The cause of these conditions is thought to be abnormalities in the smooth muscle of the urinary outflow tract (renal pelvis, ureters, or bladder) or impaired peristalsis. An elegant study by Chang et al. in this issue of the JCI describes a new mouse model of obstructive nephropathy in which the gene encoding the calcineurin B type1 isoform (Cnb1) has been deleted from mesenchyme lining the urinary outflow tract (4). These animals develop hydronephrosis due to impaired pyeloureteral peristalsis, most likely caused by a failure in the outgrowth of the renal pelvis. This new study points to the crucial role of the renal pelvis as a regulator of peristalsis in the urinary outflow tract.

\section{Effective urine transport depends on formation of proper connections between the kidney and ureters}

Development of the metanephric kidney is initiated by the ureteric bud, an epithelial sprout that forms at the base of the Wolf- 
A

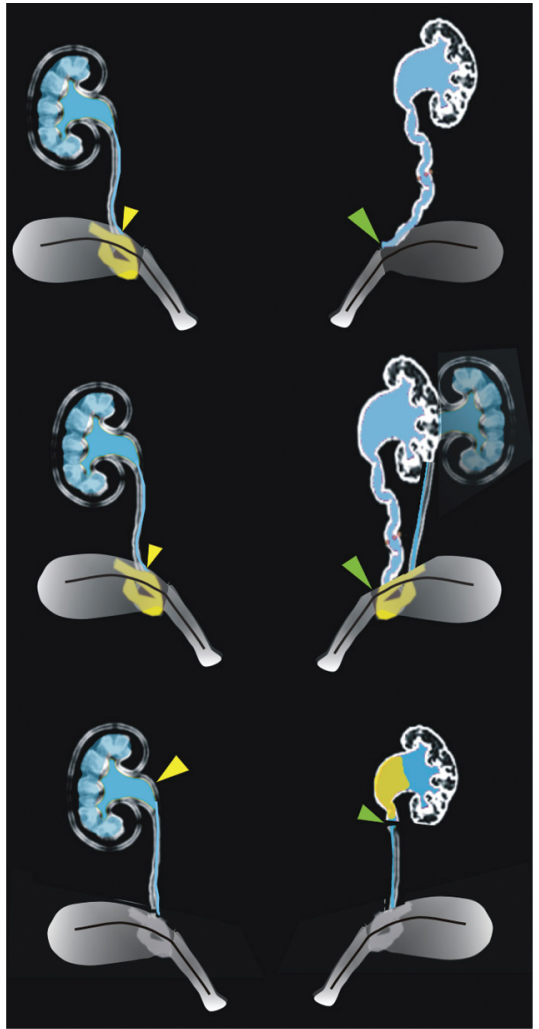

B

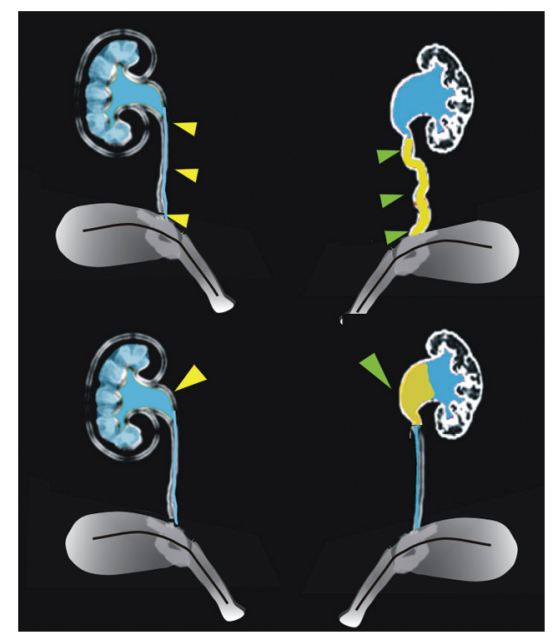

Physical obstruction

Ectopically terminating ureter in a single collecting duct system

Ectopically terminating ureter in a duplicated collecting duct system

UPJ-stenosis and atresia

Functional obstruction

Megaureter

UPJ abnormalities

fian ducts, paired epithelial tubes that regress in females and persist in males, forming a part of the genital tract. The ureteric bud invades the kidney blastema and makes contact with the metanephric mesenchyme. Once this occurs, signals from the ureteric bud induce the metanephric mesenchyme to differentiate into epithelial components of the nephron, and signals from the metanephric mesenchyme induce the ureteric bud to undergo successive rounds of branching morphogenesis, forming the renal collecting duct system. The portion of the ureteric bud that lies outside the kidney differentiates into the ureters, which are muscular tubes that conduct urine from the kidney to the bladder. The ureters are initially connected to the bladder via the Wolffian ducts, but prior to sexual differentiation, the ureter orifices undergo a maturation process, separating from the Wolffian ducts and establishing direct connections with the bladder. Expansion and downward growth of the Wolffian ducts into the primitive bladder helps induce ureter sep-
Figure 1

A schematic showing different types of obstruction that can cause hydronephrosis. (A) Top, examples of physical obstruction: ectopically terminating ureter in a single (top) or duplicated (middle) collecting duct system. In both cases the ureter joins the urinary tract outside the normal integration site in the trigone. In the example showing a duplicated system, one ureter joins normally; the other, abnormally. Bottom, uteropelvic junction (UPJ) stenosis or atresia causing physical blockage at the ureteropelvic junction. (B) Examples of functional obstruction. Top, primary megaureter caused by impaired peristalsis or defective differentiation of smooth muscle in the ureter coat. Bottom, UPJ abnormalities caused by failure in outgrowth or function of the renal pelvis. On the left, yellow filled arrowheads designate the normal structure; the abnormal structure on the right is designated by green filled arrowheads.

aration and, by an unknown mechanism, the now separated ureter orifice inserts into the base of the bladder (5). Distal ureters joining the bladder abnormally often lead to obstructed urine flow, a major cause of hydronephrosis and related disorders. A number of mouse models have been developed that display hydronephrosis linked to ectopic ureter orifices, including Gdf11 mutants (6), Bmp4 heterozygous mutants (7), Foxc1/Foxc2 mutants (8), angiotensin type 2 receptor mutants (9), and Rarab2- mutants (10).

\section{Efficient fluid transport through the urinary outflow tract depends on peristalsis}

The metanephric kidney becomes active during prenatal life, but removal of nitrogenous waste prior to birth is mediated by the placenta; thus, the volume of urine produced by the fetal kidney is relatively low. After birth, removal of nitrogenous waste shifts from the placenta to the neonatal kidney, generating an enormous increase in urine production. Once this occurs, urine must be efficiently removed from the kidney to avoid damage due to pressure buildup and toxicity. The renal pelvis is central to this process. The renal pelvis is surrounded by a thin layer of smooth muscle that forms around the renal calyces and papilla during the first weeks of life, connecting to the ureter at the ureteropelvic junction. Once a bolus of urine collects, the renal pelvis contracts, moving the urine out of the kidney into the ureters. The ureter coat contains smooth 
muscle cells that conduct peristaltic waves; thus, the ureter can undergo peristalsis independently of the renal pelvis. However, the rate and timing of peristalsis is thought to be governed by the renal pelvis, which contains "pacemaker" cells within the smooth muscle wall.

Failure in formation of the renal pelvis or impaired smooth muscle differentiation along the urinary outflow tract are a major cause of functional obstruction and hydronephrosis. Several mouse models have been developed that display functional obstruction due to a deficiency in smooth muscle lining the ureters and renal pelvis, including conditional knockouts of sonic hedgehog (11) and mutants in the gene encoding ADAMTS-1, a disintegrin and metalloproteinase with thrombospondin motifs (3). In the current issue of the JCI, Chang et al. generate a mouse line harboring a conditional knockout of the Cnb1 gene, encoding one of two calcineurin $\mathrm{B}(\mathrm{CnB})$ isoforms (4). These animals display a phenotype closely resembling obstructive nephropathy in humans. These new studies indicate that $C n b 1$ is required for outgrowth of the renal pelvis and that absence of the renal pelvis leads to functional obstruction.

\section{Calcineurin controls peristalsis by regulating outgrowth of the renal pelvis}

The present studies of Chang et al. highlight the crucial role of the renal pelvis as a modulator of peristalsis and provide a new model of obstructive nephropathy (4). Calcineurin is a calmodulin-regulated serine-threonine phosphatase required for activation of the cytoplasmic nuclear factor of activated T cells (NFATc) transcription factors. Gene-targeting studies demonstrate that the NFATc-calcineurin pathway mediates diverse functions in a number of organs and tissues. That calcineurin might have some function in the kidney was suggested by the observation that calcineurin inhibitors such as cyclosporin A, an immunosuppressive drug, can damage the kidney (12). Calcineurin is composed of a catalytic subunit (calcineurin A $[\mathrm{CnA}]$ ) and a regulatory subunit $(\mathrm{CnB})$. The $\mathrm{Cnb}$ gene encodes two isoforms, $C n b 1$ and $C n b 2$. The Cnb1 isoform is widely expressed and is required for calcineurin-NFATc signal- ing. Inactivation of the $C n b 1$ gene results in lethality at embryonic day 11 due to heart and vascular defects (13). Because mutant embryos die prior to the time at which the kidney develops, Chang et al. used mouse genetics to generate a conditional knockout in which $C n b 1$ was deleted in a subset of cells in which it was normally localized, including the mesenchyme lining the urinary outflow tract. They used two mouse lines: $C n b 1^{F}$ mice, in which Cnb1 coding sequences were flanked with loxP sites; and Pax3Cre mice, a transgenic line expressing Cre recombinase in a number of cell types, including the mesenchyme that forms smooth muscle lining the renal pelvis and ureters. In cells harboring the floxed Cnb1 allele, expression of the Pax3Cre transgene resulted in excision of Cnb1 sequences between the loxP sites and generation of a Cnb1null allele. Pax3Cre;Cnb1 $1^{F / F}$ mutants died within 3 weeks after birth. In newborn mutants, the urinary system was apparently normal; however, by postnatal day 12 , mutant kidneys had developed hydronephrosis and were severely damaged, compromising renal function.

Analysis of the mutant urinary tract by corrosive casting revealed no sign of physical obstruction, suggesting that hydronephrosis was caused by functional obstruction. Further analysis revealed that functional obstruction in Pax $3 \mathrm{Cre} ; \mathrm{Cnb} 1^{\mathrm{F} / \mathrm{F}}$ kidneys was likely caused by impaired peristalsis as a consequence of failure in renal pelvis formation. The authors demonstrate that $C n b 1$ regulates pelvis outgrowth by controlling proliferation of the mesenchyme that forms the smooth muscle lining of the renal pelvis. Interestingly, mutant ureters still underwent peristalsis in the absence of the renal pelvis, but peristaltic contractions were abnormal, suggesting that the renal pelvis might exert more control than previously thought on downstream peristaltic waves propagated thought the ureter. The presence of similar defects in knockout mice lacking the angiotensin type 1 receptor suggests that calcineurin-NFAT signaling may be important for angiotensin signaling, or vice versa. The availability of mouse models of obstructive nephropathy together with powerful genomic techniques will be of great importance in understanding the etiology of lifethreatening conditions such as obstructive nephropathy and will help to identify genetic pathways normally important in urinary tract formation and function.

\section{Acknowledgments}

The author would like to thank Helen Liapis for critical reading of the manuscript.

Address correspondence to: Cathy Mendelsohn, Departments of Urology and Pathology, Columbia University College of Physicians and Surgeons, 650 West 168th Street, BB 1502, New York, New York 10032, USA. Phone: (212) 305-1591; Fax: (212) 305-6851; E-mail: clm20@columbia.edu.

1. Chevalier, R.L. 1998. Pathophysiology of obstructive nephropathy in the newborn. Semin. Nephrol. 18:585-593.

2. Woolf, A.S., Winyard, P.J.D., Hermanns, M.M., and Welham, J.M. 2003. Maldevelopment of the human kidney and lower urinary tract: an overview. In The kidney: from normal development to congenital disease. P.D. Vize, A.S. Woolf, and J.B.L. Bard, editors. Academic Press Inc. Amsterdam, The Netherlands/ Boston, Massachusetts, USA. 377-393.

3. Kuwayama, F., Miyazaki, Y., and Ichikawa, I. 2002. Embryogenesis of the congenital anomalies of the kidney and the urinary tract. Nephrol. Dial. Transplant. 17:45-47.

4. Chang, C.-P., et al. 2004. Calcineurin is required in urinary tract mesenchyme for the development of the pyeloureteral peristaltic machinery. J. Clin. Invest. 113:1051-1058. doi:10.1172/JCI200420049.

5. Batourina, E., et al. 2001. Vitamin A controls epithelial/mesenchymal interactions through Ret expression. Nat. Genet. 27:74-78.

6. Esquela, A.F., and Lee, S.J. 2003. Regulation of metanephric kidney development by growth/differentiation factor 11. Dev. Biol. 257:356-370.

7. Miyazaki, Y., Oshima, K., Fogo, A., Hogan, B.L., and Ichikawa, I. 2000. Bone morphogenetic protein 4 regulates the budding site and elongation of the mouse ureter. J. Clin. Invest. 105:863-873.

8. Kume, T., Deng, K., and Hogan, B.L. 2000. Murine forkhead/winged helix genes Foxc1 (Mf1) and Foxc2 (Mfh1) are required for the early organogenesis of the kidney and urinary tract. Development. 127:1387-1395.

9. Nishimura, H., et al. 1999. Role of the angiotensin type 2 receptor gene in congenital anomalies of the kidney and urinary tract, CAKUT, of mice and men. Mol. Cell. 3:1-10.

10. Mendelsohn, C., et al. 1994. Function of the retinoic acid receptors (RARs) during development (II). Multiple abnormalities at various stages of organogenesis in RAR double mutants. Development. 120:2749-2771.

11. Yu, J., Carroll, T.J., and McMahon, A.P. 2002. Sonic hedgehog regulates proliferation and differentiation of mesenchymal cells in the mouse metanephric kidney. Development. 129:5301-5312.

12. Wilkinson, A. 2001. Progress in the clinical application of immunosuppressive drugs in renal transplantation. Curr. Opin. Nephrol. Hypertens. 10:763-770.

13. Graef, I.A., Chen, F., and Crabtree, G.R. 2001. NFAT signaling in vertebrate development. Curr. Opin. Genet. Dev. 11:505-512. 